\title{
Solid Self-Microemulsifying Drug Delivery System (SMEDDS) of Primaquine: Bio-distribution and Enhanced Liver Uptake
}

Piyush Chudiwal* and Swaroop Lahoti

Department of Pharmaceutical Sciences and Technology, Y B Chavan College of Pharmacy, Aurangabad, India

\begin{abstract}
Aim: The present research work was aimed at developing a self-microemulsifying drug delivery system (SMEDDS) of Primaquine $(P Q)$ with increased liver uptake and hence enhanced antimalarial efficacy against the liver stages of Plasmodium vivax.
\end{abstract}

Materials and methods: SMEDDS was formulated using generally regarded as safe (GRAS) excipients and adsorbed on Aerosil 200. The optimized SMEDDS were characterized for various physicochemical parameters. Pharmacodynamic efficacy in murine model was evaluated using Peter's Four Day Suppresive Test. Biodistribution studies were carried out using flow cytometry.

Results: The adsorbed SMEDDS showed a particle size of $75 \mathrm{~nm}$ and exhibited enhanced antimalarial efficacy as compared to marketed formulations. Biodistribution studies revealed enhanced uptake in the liver.

Conclusion: Preliminary studies in lower animals indicated the potential of SMEDDS to enhance the uptake of PQ at its site of action i.e. liver.

Keywords: Pharmacodynamic efficacy; Biodistribution; Liver uptake; SMEDDS; Primaquine

\section{Introduction}

Malaria is a major cause of illness and death in children and adults, especially in tropical countries. The situation has recently become even more serious due to the increase in resistance to the drugs normally used to combat the parasites that cause the disease. In humans, malaria is caused by five distinct species of the blood borne Apicomplexan parasite Plasmodium: P. vivax, $P$. falciparum, $P$. malariae, $P$. Ovale and P. knowelsi [1]. P. vivax and P. ovale persist in the liver in the form of hypnozoites for years and can cause an erythrocytic infection upon reactivation $[2,3]$. Primaquine (PQ), an 8 -aminoquinoline drug, has been an important antimalarial agent for over 40 years because of its unique effectiveness against exoerythrocytic forms of both $P$. vivax and $P$. ovale $[4,5]$. PQ is useful to fight malaria on three different fronts: (i) primary prophylaxis against all species of malaria, (ii) presumptive antirelapse therapy (terminal prophylaxis) for persons extensively exposed to $P$. vivax or $P$. ovale, (iii) radical cure in individuals infected with $P$. vivax or $P$. ovale. In endemic regions, $P Q$ is used as a gametocytocide to prevent the transmission of the infection from the human host to the mosquitoes, thus blocking the spread of the disease. It is the only anti-malarial effective in treating the liver stages of the parasite [6]. However PQ is characterized by dose-limiting side effects like acute hemolytic anemia in patients with G6PD deficiency, methanoglobenemia, leukocytopenia, leukocytosis, GI disturbances and abdominal cramps $[7,8]$. Further, PQ is also characterized by low plasma half-life $(5.6 \mathrm{~h})$ and is subject to first-pass metabolism, which requires frequent administration and amplifies its adverse effects [9]. In view of its crucial role in antimalarial chemotherapy, it is important to reduce the adverse effects of PQ. To improve the therapeutic efficacy of the drug and diminish its toxicity, many researchers have attempted to encapsulate PQ into drug delivery systems, such as liposomes, microspheres, and nanoparticles, or to conjugate $\mathrm{PQ}$ to protein carriers to modify its toxicity profile [10]. Targeting PQ to its site of action, i.e., the liver is likely to reduce the therapeutic dose and result in a toxicity reduction. However, $\mathrm{PQ}$ is advised not to be administered parenterally because of the risk of marked hypotension. Thus the oral route is preferable for PQ administration. Targeting via oral route is made possible by using a nanoparticulate system that mimics natural lipoproteins with regards to the intestinal absorption and distribution [1].

SMEDDS is defined as isotropic mixture of oil, surfactant, cosurfactant, and drug that rapidly forms o/w microemulsion when exposed to aqueous media under conditions of gentle agitation or digestive motility that is encountered in GI tract. SMEDDS presents the drug in nanosized droplets offering large interfacial area for drug diffusion [11]. SMEDDS can be easily scaled up and are commercially feasible. They can also be converted to solid dosage forms and filled in hard gelatin capsules and show improved physical stability upon long term storage due to their anhydrous nature [12]. Self-microemulsifying system of PQ would be an efficient, and convenient and more likely to have higher patient compliance. Hence, the feasibility of SMEDDS as a strategy for improving the delivery of PQ was evaluated. PQ free base, which exists in liquid state at room and physiologic temperature, served as an oily phase of the microemulsions in the present investigation.

\section{Materials and Methods}

\section{Chemicals}

Primaquine Diphosphate was a generous gift from Ipca Laboratories Limited, Mumbai. Cremophor EL (PEG - 35 Castor oil), Cremophor RH 40 (Polyoxyl 40 Hydrogenated Castor Oil), Solutol HS

*Corresponding author: Piyush Chudiwal, Department of Pharmaceutical Sciences and Technology, Y B Chavan College of Pharmacy, Aurangabad-431003, Maharashtra, India, Tel: 9102402381129; E-mail: piyush_chudiwal@yahoo.co.in

Received: February 09, 2018; Accepted: February 20, 2018; Published: February 26, 2018

Citation: Chudiwal P, Lahoti S (2018) Solid Self-Microemulsifying Drug Delivery System (SMEDDS) of Primaquine: Bio-distribution and Enhanced Liver Uptake. J Nanomed Nanotechnol 9: 483. doi: 10.4172/2157-7439.1000483

Copyright: (C) 2018 Chudiwal P, et al. This is an open-access article distributed under the terms of the Creative Commons Attribution License, which permits unrestricted use, distribution, and reproduction in any medium, provided the original author and source are credited. 
15 (Polyethyleneglycol-15-hydroxystearate) (BASF, Mumbai, India), Gelucire 44/14 (Lauroyl macrogol-32 glycerides), (Gattefosse, France), Labrasol 9 Saturated poly glycolised C8-C10 glycerides) (Colorcon Asia Mumbai, India) were obtained as gift samples. Tween 20 (Polyoxyethylene sorbitan monolaurate), Tween 80 (Polyoxyethylene sorbitan monooleate) (S D Fine-Chemicals Limited, Mumbai, India) were purchased. Lactose (Signett, Mumbai), microcrystalline cellulose and Aerosil 200 (Signet, Mumbai), Neusilin (Aluminium metasilicate Gangwal Chemicals, Mumbai) were obtained as gift samples. All organic solvents (AR grade) were purchased from S D Fine-Chemicals, Limited, Mumbai, India. Hard gelatin capsules (Associated Capsules Group, Mumbai, India) were obtained as gift samples. All excipients and reagents were used as received. Freshly prepared double distilled water was used.

\section{Parasite}

Plasmodium berghei ANKA strain was used for in vivo evaluation of antimalarial activity. The strain was free of contamination with Eperythrozoon coccoides. The strain is well characterized and it is known to result in high mortality in mice, providing a good model to estimate survival and antimalarial efficacy. It is sensitive to all antimalarial agents currently in use.

\section{Animals}

Animal experiments were carried out according to the CPCSEA (Committee for the purpose of the control and supervision on experiments on animals) guidelines. In house bred, eperythrozoon free male Swiss albino mice aged 2-4 weeks having body weight in the range of 30-45 g were used for the study. The animals, held at a temperature of $22 \pm 3^{\circ} \mathrm{C}$ and $65 \%$ relative humidity were fed a standard mouse diet and provided with clean drinking water ad libitum throughout the experiments.

\section{Release of PQ free base and its characterization}

PQ free base was released according to the reported method in the literature [9]. Briefly, Primaquine Diphosphate (3.0 g) was dissolved in about $100 \mathrm{ml}$ of distilled water and the $\mathrm{pH}$ was increased to 12.0 with ammonia solution. The released free base was extracted twice with $100 \mathrm{ml}$ of chloroform. The organic phase was washed twice with water and twice with a saturated solution of sodium chloride. Before evaporation, the chloroform was dried by passing through anhydrous sodium sulphate. PQ obtained in this way was confirmed by infrared spectroscopy (Perkin Elmer - FTIR Spectrophotometer, Spectrum RXI).

\section{Screening of surfactants for self-microemulsifying ability}

Self-microemulsification ability of surfactants was assessed to select the best surfactant from a large pool of surfactants. The selfmicro emulsification properties of the surfactants were determined by mixing surfactants with $\mathrm{PQ}$ at $15 \% \mathrm{w} / \mathrm{w}$ and, vortexing to form isotropic and homogenous mixture. Various cosurfactants were screened by mixing with selected surfactants in $\mathrm{K}_{\mathrm{m}}$ ratios $(\mathrm{Km}$ ratio is mass ratios of surfactant to co-surfactant) of $0.5,0.75,1.0,1.25$,
1.5 and 2.0 (Data for $\mathrm{K}_{\mathrm{m}} 1$ shown). About $50 \mathrm{mg}$ of oil-surfactantcosurfactant mixture was dispersed into $50 \mathrm{ml}$ of double distilled water in a glass beaker with gentle stirring. Visual test was used to assess the self-microemulsification properties of the surfactants in terms of dispersibility, ease of self-emulsification, and final appearance as explained in Table 1.

\section{Construction of pseudoternary phase diagrams}

The boundaries of the microemulsion (ME) domains were determined with the aid of pseudoternary phase diagrams. Phase diagrams were prepared by water titration method. Series of blends of the oil phase and surfactant- cosurfactant mixture ranging from ratio of 00:10 to 10:00 were placed in preweighed test tubes. Water was added dropwise to the resultant mixtures. After each addition, the mixtures were vortexed for 2-3 $\mathrm{min}$, and were allowed to equilibrate at room temperature for $15 \mathrm{~min}$. After equilibration, the mixtures were examined visually for transparency. The point at which the mixture became turbid or showed signs of phase separation was considered as end point of the titration. The area of microemulsion existence was determined and denoted as ME.

\section{Effect of oil content on mean particle size}

The oil content was varied from $2045 \%$ w/w. Formulations equivalent to $15 \mathrm{mg} \mathrm{PQ}$ were weighed and diluted in water as discussed for self-microemulsification ability studies. The dispersions were observed visually for optical clarity and subjected to particle size analysis using Zetasizer Nano ZS (Malvern Instruments, UK). The particle size was determined immediately after their formation. To assess physical stability of resulting aqueous dispersion on large dilutions, the dispersions were kept at room temperature for six hours and the particle size was measured again.

\section{Preparation of SMEDDS}

PQ SMEDDS were prepared by mixing accurately weighed PQ and surfactant/- cosurfactant in a glass beaker. Components were mixed and heated to $45^{\circ} \mathrm{C}$ to facilitate uniform mixing to form a homogenous mixture. For the preparation of solid SMEDDS, PQ SMEDDS was mixed with anhydrous lactose, microcrystalline cellulose, VBP -2, Aerosil 200, and Neusilin in various ratios (2:1, 1:1, 1:2). Briefly, PQ SMEDDS was added dropwise over the solid adsorbent contained in a broad bottom beaker. After each addition, the mixture was homogenized using glass rod to ensure uniform distribution of the droplet. The adsorbent that was required in a small amount to give a free flowing solid microemulsion preconcentrate with high bulk density was chosen for further studies.

\section{Particle size analysis}

Robustness to dilution and effect of $\mathrm{pH}$ of dilution medium on the particle size was studied by diluting formulations, equivalent to $15 \mathrm{mg}$ of PQ with $250 \mathrm{ml}$ of different dilution media (double distilled water, buffers at $\mathrm{pH} 1.2,3.5,4.5,6.8$ and 8.0). The mean droplet size and size distribution of resulting dispersions were determined immediately after their formation. The resulting dispersions were then allowed to stand for

\section{Dispersibility}

Oil droplets spread rapidly in water forming clear and transparent microemulsion

Formulation formed transparent, gel like intermediate structure prior to dispersing completely but could form microemulsion 
six hours at room temperature to assess dilution stability. Formulations were analyzed for particle size again. The particle size measurements were made for both unadsorbed and adsorbed SMEDDS. The adsorbed systems were filtered through $0.45 \mu \mathrm{m}$ membrane before measurement.

\section{Drug content}

PQ content in the SMEDDS formulation was determined by using an in house developed HPLC method. The HPLC system consisted of Jasco PU 2080 Plus Intelligent HPLC Pump, Jasco, Japan, equipped with Hibar250-4,6, Purospher Star RP-18e column and a Jasco UV 2075 Intelligent UV-VIS Detector, Jasco, Japan, with a Rheodyne 7725 injector USA managed by Jasco Borwin Chromatography software version 1.05. The mobile phase (Methanol:Acetonitrile: Phosphate Buffer $\mathrm{pH}$ 3.0, 27:23:50) was run at a flowrate of $1 \mathrm{ml} / \mathrm{min}$ and detection of PQ was carried out at $265 \mathrm{~nm}$. Amount of SMEDDS equivalent to 5 $\mathrm{mg}$ of PQ were weighed accurately and transferred to $50 \mathrm{ml}$ volumetric flasks. Volume was made up to $50 \mathrm{ml}$ with methanol in volumetric flask to obtain a stock solution of $100 \mu \mathrm{g} / \mathrm{ml}$. Stock solution was then diluted suitably to obtain solution of concentration $5 \mu \mathrm{g} / \mathrm{ml}$. The solution was then injected in HPLC system so as to determine drug content. All the experiments were carried out in triplicate for all the PQ formulations. Also the stability of PQ in various buffers viz. $\mathrm{pH} 1.2,3.5,4.5,6.8$ and 8.0 was determined by HPLC.

\section{Evaluation of flow properties}

Flow properties of solid formulations are determined to ascertain their behaviour during further processing like capsule filling and tabletting. Flow properties such as angle of repose, Hausner ratio and Carr's index along with density were assessed for the adsorbed PQ SMEDDS [13].

\section{Disintegration time}

The test was carried out as per Indian Pharmacopoeia 1996 in appendix 7 (7.1 disintegration test for tablets and capsules). Disintegration was carried out using Electrolab Disintegration test apparatus. Disintegration time for capsules filled with respective formulations was determined by placing six capsules, one in each tube, in disintegration apparatus using distilled water maintained at $37 \pm$ $0.5^{\circ} \mathrm{C}$ as a media. The time required for complete passing of capsule fraction except if any fragments of capsule shells from mesh10 \# to media was taken as disintegration time.

\section{In vitro dissolution profile}

In vitro release of PQ SMEDDS was studied using USP XXIII Apparatus I at $37 \pm 0.50^{\circ} \mathrm{C}$ at $50 \mathrm{rpm}$ in dissolution media namely, $0.01 \mathrm{~N} \mathrm{HCl}$ and $\mathrm{pH} 6.8$ phosphate buffer. The volume of dissolution medium was $500 \mathrm{ml}$. During the study, $5 \mathrm{ml}$ of aliquots were removed at predetermined time intervals $(15,30,45$, and $60 \mathrm{~min}$.) from the dissolution medium and replaced with fresh medium. The amount of PQ released in the dissolution medium was determined using UV Spectrophotometry at $\lambda_{\max }=265 \mathrm{~nm}$.

\section{Stability studies}

Chemical and physical stability of PQ SMEDDS was assessed at various storage conditions viz. $25 \pm 2^{\circ} \mathrm{C} / 60 \pm 5 \% \mathrm{RH}, 30 \pm 2^{\circ} \mathrm{C} / 65 \pm 5 \%$ $\mathrm{RH}, 40 \pm 2^{\circ} \mathrm{C} / 75 \pm 5 \% \mathrm{RH}$ as per ICH guidelines. SMEDDS equivalent to $15 \mathrm{mg}$ PQ was filled in size 2 hard gelatin capsules and packed in aluminium strips and stored at aforementioned storage conditions up to 3 months. Samples were removed at $0,30,60,90$, and 180 days of interval and were assessed for content of $\mathrm{PQ}$, mean globule size and polydispersity index, and in vitro dissolution profile.

\section{In vivo efficacy studies}

"The protocol of the study was approved by Institutional Animal Ethics Committee, Y. B. Chavan College of Pharmacy, Aurangabad; vide protocol approval no:, (CPCSEA/IAEC/P'COL-08/2015-16/21), The Declaration of Helsinki as amended in Seoul 2008 for humans, and the European Community guidelines as accepted principles for the use of experimental animals, were adhered to. All the experimental procedures were carried out as per Committee for the Purpose of Control and Supervision of Experiments on Animals (CPCSEA) guidelines released by Ministry of Environment, Forests and Climate Change, Govt. of India."

The protocol for "Peters four day suppressive test" as described by Peters et al. was used for the study [14]. Male Swiss mice free of mycoplasma were infected by intraperitoneal inoculation of donor mouse blood diluted in ACD (acid citric dextrose) containing approximately $10^{6}$ infected RBCs on day ' 0 '. Starting from day ' 0 ' to day ' 3 ' post infection, the different groups of mice were given PQ formulations by oral gavage. The summary of the treatment groups is given in Table 2. The extent of parasitemia was determined from Giemsa-stained thin blood smears prepared from tail blood at different time points as indicated. Parasitemia was reported as percentage parasitemia after counting 1000 RBCs from each slide. Activity was calculated by the following formula suggested in the standard protocol by Fidlock et al. [15]. The numbers of surviving animals on day 30 of the experiment were noted.

Percent Activity $=100-\{$ Mean Parasitemia of treated group/Mean Parasitemia of Control group $\} \times 100$.

\section{Biodistribution studies}

Biodistribution studies of PQ SMEDDS were carried using flow cytometry. Rhodamine B dissolved in methanol was added to PQ free base as the fluorescent marker. The SMEDDS of PQ were prepared using the fluorescent labelled PQ free base. For PQ suspension group, fluorescently labelled PQ free base was dispersed in purified water. Rhodamine B was used in a final concentration of $0.02 \mathrm{mM}$. A single dose of fluorescent labelled formulation was administered orally in the mice. Mice were sacrificed at various time points $(0.25,0.5,1,2,4,8,12$, 24 , and $48 \mathrm{~h}$ ) to collect tissues and organs viz. the spleen, the thymus, the liver, the kidneys, the lungs, the draining lymph node, the brain, bone marrow and blood. The organs were homogenised and single cell suspensions prepared by passing through a $0.40 \mu \mathrm{m}$ cell strainer. Erythrocytes were removed by lysis using ACK buffer $\left(0.15 \mathrm{M} \mathrm{NH}_{4} \mathrm{Cl}\right.$, $10 \mathrm{mM} \mathrm{KHCO}, 0.1 \mathrm{mM} \mathrm{Na}$ EDTA), the cells were washed thrice with PBS, and analysed by flow cytometry using Guava PCA 96 flow cytometer. The results were expressed as mean fluorescence intensity $\mathrm{v} / \mathrm{s}$ time. The intensity of fluorescence was directly proportional to the PQ concentration in the tissues.

\begin{tabular}{|c|c|c|}
\hline Group & Infection & Treatment \\
\hline $\mathrm{A}$ & + & Control \\
\hline $\mathrm{B}$ & + & Placebo SMEDDS \\
\hline $\mathrm{C}$ & + & PQ Suspension $15 \mathrm{mg}$ \\
\hline $\mathrm{D}$ & + & Marketed Formulation $7.5 \mathrm{mg}$ \\
\hline $\mathrm{E}$ & + & Marketed Formulation $15 \mathrm{mg}$ \\
\hline $\mathrm{F}$ & + & PQ SMEDDS $7.5 \mathrm{mg}$ \\
\hline $\mathrm{G}$ & + & PQ SMEDDS $15 \mathrm{mg}$ \\
\hline & \multicolumn{3}{|c|}{ Table 2: Summary of treatment groups. } \\
\hline
\end{tabular}




\section{Statistical analysis}

One-way ANOVA followed by Dunnett's test was applied using GraphPad Prism 5 Demo version, for ascertaining statistical significances. Differences were considered significant at ${ }^{*} \mathrm{p}<0.05$. In in vivo efficacy studies, all values are expressed as Mean $\pm \mathrm{SD}$, $\mathrm{n}=8$, wherein all treated groups were compared with PQ marketed formulation group. In biodistribution studies, all values are expressed as Mean $\pm S D, n=3$, wherein PQ SMEDDS group was compared with PQ suspension group.

\section{Results and Discussion}

\section{Release of $P Q$ free base and its characterization}

Since the basic form of PQ is a dark brown viscous oily liquid (boiling point $177^{\circ} \mathrm{C}$ ) and is also more lipophilic than its salt form, it was decided to use PQ base as the oil phase. This could be of particular advantage in terms of achieving higher drug loading and increased partitioning to the liver. The infrared (IR) spectra of the released base matched with the standard spectra as per Indian Pharmacopoeia. N-H bending and stretching vibrations that characterized free amino group were seen at 1519.2 and $3386.1 \mathrm{~cm}^{-1}, \mathrm{~N}-\mathrm{H}$ wagging was observed at $752.8 \mathrm{~cm}^{-1}$ indicating liquid primary and secondary amine whereas aliphatic C-H linkage was characterized at $1216.0,1595.5 \mathrm{~cm}^{-1}$.

\section{Screening of surfactants for self-microemulsifying ability}

Self-microemulsification ability of the surfactants was determined by mixing surfactants with PQ and dispersing the resultant mixture in double distilled water. Grading for emulsification of surfactants is shown in Table 3 which clearly distinguished ability of surfactants to emulsify PQ. The study indicated that Cremophors had very good ability to emulsify PQ followed by Tween 20 and Tween 80, whereas, Labrasol, Lutrol F68, and Lutrol F 127 was found to be very poor emulsifiers. Cremophor RH 40 was found to be the best emulsifier for PQ. Nevertheless, Cremophor EL and Tweens were also found work well. In addition to their ability to solubilize poorly soluble drugs, non-ionic surfactants are also known to be less irritant and cytotoxic than their anionic and cationic counterparts; and are less affected by $\mathrm{pH}$ and changes in ionic strength that are likely to be encountered in gastrointestinal environment As shown in Table 4, all the combinations of surfactant mixtures formed stable clear microemulsions. Hence, Cremophors, Tweens and Gelucire 44/14 were therefore selected for further study.

\section{Construction of pseudoternary phase diagrams}

Pseudoternary phase diagrams were constructed by water titration method. The phase behavior of all the systems was studied at a fixed ratio of surfactant to cosurfactant $1: 1$, as it was found to be optimum

\begin{tabular}{|l|c|c|}
\hline Surfactant & $\begin{array}{c}\text { Observation with } \mathbf{P Q} \text { as } \\
\text { oil phase }\end{array}$ & $\begin{array}{c}\text { Observation at the end } \\
\text { of } \mathbf{2 4} \mathbf{~}\end{array}$ \\
\hline Cremophor RH 40 & $\mathrm{ME}$ & $\mathrm{ME}$ \\
\hline Solutol HS 15 & $\mathrm{E}$ & $\mathrm{E}$ \\
\hline Cremophor EL & $\mathrm{ME}$ & $\mathrm{ME}$ \\
\hline Gelucire 44/14 & $\mathrm{ME}$ & $\mathrm{ME}$ \\
\hline Tween 80 & $\mathrm{ME}$ & $\mathrm{ME}$ \\
\hline Tween 20 & $\mathrm{ME}$ & $\mathrm{ME}$ \\
\hline Labrasol & $\mathrm{PE}$ & $\mathrm{PE}$ \\
\hline Lutrol F 68 & $\mathrm{PE}$ & $\mathrm{PE}$ \\
\hline Lutrol F 127 & $\mathrm{PE}$ & $\mathrm{PE}$ \\
\hline
\end{tabular}

Table 3: Emulsification efficiency of various surfactants.

\begin{tabular}{|c|c|c|}
\hline $\begin{array}{c}\text { Surfactant-cosurfactant } \\
\text { combinations }\end{array}$ & $\begin{array}{c}\text { Observation with PQ } \\
\text { as oil phase }\end{array}$ & $\begin{array}{c}\text { Observation at the } \\
\text { end of } \mathbf{2 4} \mathbf{~ h}\end{array}$ \\
\hline Cremophor RH 40+Gelucire 44/14 & ME & ME \\
\hline Solutol HS $15+$ Gelucire 44/14 & ME & ME \\
\hline Cremophor EL+Gelucire 44/14 & ME & ME \\
\hline Tween $80+$ Gelucire 44/14 & ME & ME \\
\hline Tween $20+$ Gelucire 44/14 & ME & ME \\
\hline Tween $80+$ Tween 20 & ME & ME \\
\hline
\end{tabular}

Table 4: Emulsification efficiency of surfactant - cosurfactant combinations.

for all the systems during preliminary investigations (data not shown). The phase diagrams (Figure 1) have two distinct regions viz. ME region and non-ME region. The ME region represents clear optically isotropic microemulsion area and non-ME region represents the turbid dispersed systems identified based on visual observation. Their properties were further not characterized in detail for any of the investigated systems. The clear microemulsion region is represented as ME region in the phase diagrams. As evident from the pseudoternary phase diagrams, both the surfactant combinations gave a similar microemulsion region. Also, all the combinations were infinitely dilutable at higher surfactant concentrations. Since preliminary data suggested Cremophor RH 40 as the best emulsifier for PQ and Gelucire 44/14 is known to be an absorption enhancer, this combination was selected for further study.

\section{Effect of oil content on mean globule size}

Effect of oil content was studied by varying the percent of oil loading keeping the other constituents constant. The systems were dispersed in double distilled water and their particle size was measured. Effect of oil loading on mean globule size is shown in Figure 2. An increase in the oil content upto $40 \% \mathrm{w} / \mathrm{w}$ of oil surfactant mixture did not affect the particle size significantly and was found to be less than $35 \mathrm{~nm}$ even when allowed to stand for 6 hours. A sudden increase in particle size was observed at $45 \% \mathrm{w} / \mathrm{w}$ of oil surfactant mixture which further increased after 6 hours. Hence, $40 \%$ w/w oil loading was optimized for the final formulation.

\section{Selection of optimum composition}

The optimized formulation was selected based on the drug loading efficiency and consistency in mean globule size at varying $\mathrm{pH}$. Of all the adsorbents tried, Aerosil 200, VBP - 2, Neusilin could adsorb PQ SMEDDS efficiently and gave free flowing powder. In the preliminary stability studies, Aerosil 200 gave stable sytems and hence was selected for further evaluation. The selected components were as follows:

- Oil Phase: PQ

- Surfactant/Cosurfactant: Cremophor RH 40/Gelucire 44/14

- Adsorbent: Aerosil 200.

\section{Particle size analysis}

The particle size of the PQ SMEDDS was determined by dispersing the SMEDDS in a fixed volume of water. Also the physical stability of the SMEDDS was determined by dispersing the SMEDDS in buffers of various $\mathrm{pH}$ and measuring their particle size. The particle size of the PQ SMEDDS before and after adsorption, and in different $\mathrm{pH}$ media is depicted in Table 5. Physical integrity of the microemulsion formed and drug solubilization capacity after dilution of SMEDDS must be assessed and ensured as it gives an idea about its performance in vivo. In view of this, PQ SMEDDS were diluted with aqueous phases differing in $\mathrm{pH}$. PQ SMEDDS dispersed effectively and formed microemulsion in a minute without precipitation of the drug. The optimized PQ 


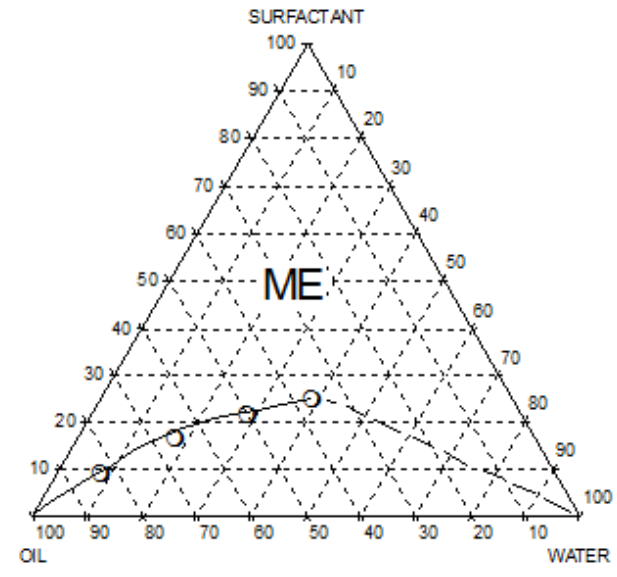

(a) Cremophor RH 40 and Gelucire 44/14

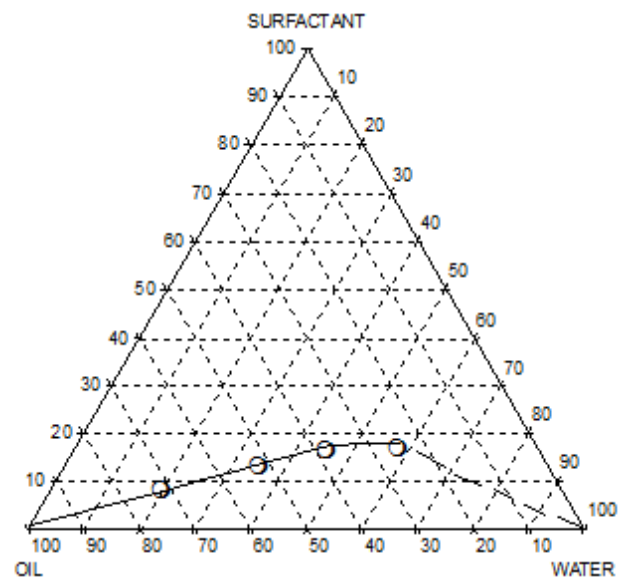

(c) Tween 80 and Gelucire $44 / 14$

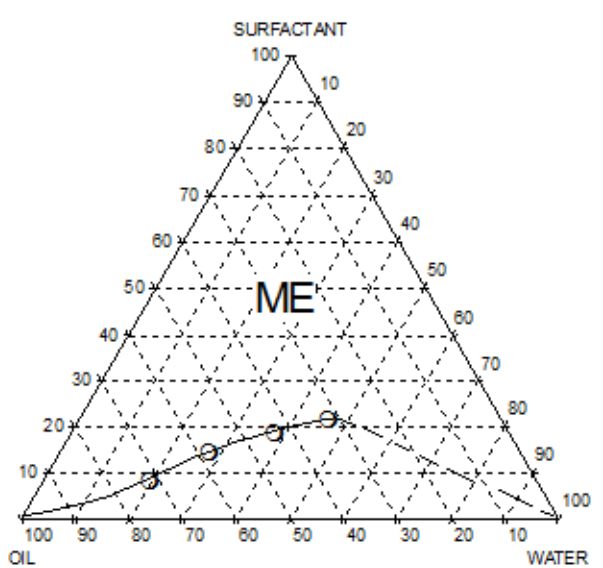

(b) Cremophor EL and Gelucire 44/14

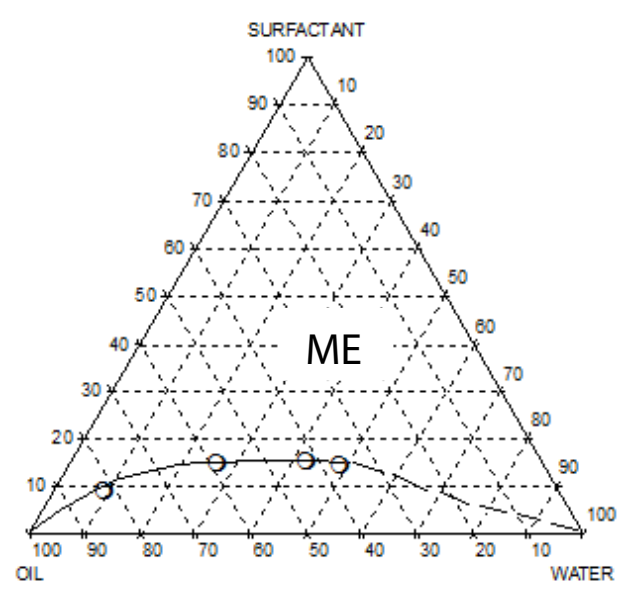

(e) Tween 80 and Tween 20

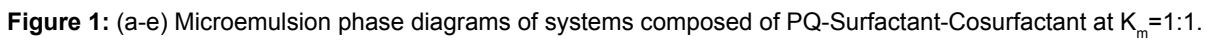



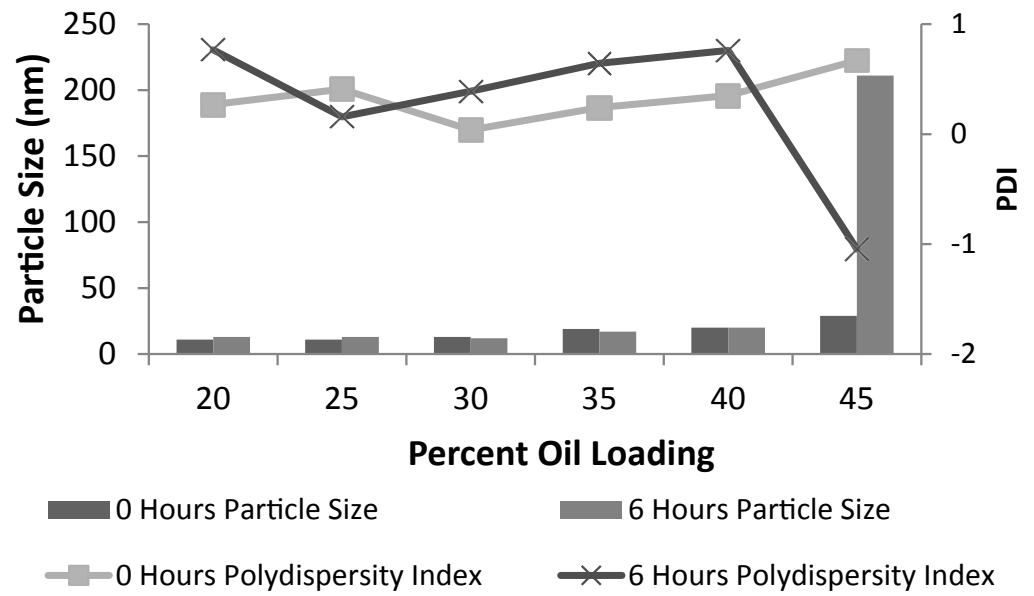

Figure 2: Effect of oil loading on particle size and polydispersity index (PDI) of the system containing Cremophor $\mathrm{RH} 40+\mathrm{Gelucire} 44 / 14$.

\begin{tabular}{|c|c|c|c|c|c|c|c|c|}
\hline \multirow[t]{3}{*}{ Dilution Phase } & \multicolumn{4}{|c|}{ Unadsorbed SMEDDS } & \multicolumn{4}{|c|}{ Adsorbed SMEDDS } \\
\hline & \multicolumn{2}{|r|}{0 Hours } & \multicolumn{2}{|r|}{6 Hours } & \multicolumn{2}{|r|}{0 Hours } & \multicolumn{2}{|c|}{6 Hours } \\
\hline & $\begin{array}{l}\text { Particle Size } \\
\text { (nm) }\end{array}$ & Polydispersity Index & $\begin{array}{c}\text { Particle Size } \\
\text { (nm) }\end{array}$ & Polydispersity Index & $\begin{array}{l}\text { Particle Size } \\
\text { (nm) }\end{array}$ & Polydispersity Index & $\begin{array}{c}\text { Particle Size } \\
\text { (nm) }\end{array}$ & $\begin{array}{l}\text { Polydispersity } \\
\text { Index }\end{array}$ \\
\hline Water & 22 & 0.423 & 22 & 0.236 & 75 & 0.358 & 72 & 0.325 \\
\hline Buffer pH 1.2 & 24 & 0.356 & 21 & 0.164 & 77 & 0.314 & 78 & 0.321 \\
\hline Buffer pH 3.5 & 22 & 0.41 & 24 & 0.106 & 75 & 0.351 & 77 & 0.356 \\
\hline Buffer pH 4.5 & 23 & 0.308 & 23 & 0.241 & 78 & 0.301 & 77 & 0.331 \\
\hline Buffer pH 6.8 & 22 & 0.32 & 23 & 0.076 & 78 & 0.347 & 74 & 0.341 \\
\hline Buffer pH 8.0 & 24 & 0.274 & 25 & 0.254 & 76 & 0.361 & 72 & 0.352 \\
\hline
\end{tabular}

Globule size expressed as mean, $(n=3)$, where relative standard deviation was $<10 \%$.

Table 5: Particle size and Polydispersity index of PQ SMEDDS.

formulations showed an increase in globule size after adsorbing it onto solid carrier. This could probably be due to some alteration in properties of PQ SMEDDS that might have occurred due to its intense mixing with solid carrier in the process of adsorption.

\section{Drug content}

The drug content of PQ SMEDDS was determined by an inhouse developed and validated HPLC method. The PQ content in the adsorbed formulation was found to be $99.01 \pm 2.15$ percent.

\section{Flow properties}

Flow properties of solid formulations were determined to ascertain their behaviour during further processing like capsule filling and tabletting. The angle of repose less than $25^{\circ}$ indicates good flow. Carr's Index is indicative of the flow properties of the material. An index of below 15 indicates good flow properties. A Hausner ratio of less than 1.25 indicates good flow and spherical particles. The summary of various parameters determined to evaluate the flow properties of PQ SMEDDS is shown in Table 6. The results indicate the adsorbed SMEDDS had good flow properties and was found to be suitable for capsule filling.

\section{Disintegration time}

The disintegration time was determined using a disintegration apparatus. The disintegration time of the capsules was found to be 1 minute. Disintegration determines the time required for the formulations to come in contact with the gastric fluids. Fast disintegration of the capsules is necessary as it can influence the release

\begin{tabular}{|c|c|}
\hline Property & SMEDDS \\
\hline Bulk Density $(\mathrm{gm} / \mathrm{ml})$ & 0.51 \\
\hline Tapped Density $(\mathrm{gm} / \mathrm{ml})$ & 0.65 \\
\hline Angle of Repose & $17^{\circ}$ \\
\hline Carr's Index $(\%)$ & 14 \\
\hline Hausner's ratio & 1.13 \\
\hline
\end{tabular}

Table 6: Summary of the flow properties of PQ SMEDDS.

of drug from the dosage forms and hence can affect the bioavailability.

\section{In vitro dissolution profile}

The in vitro release of $\mathrm{PQ}$ from the SMEDDS formulation was determined using an USP XXIII dissolution apparatus. The recommended dissolution medium for Primaquine diphosphate in USP 30-NF25 is $0.01 \mathrm{~N} \mathrm{HCl}$. In vitro dissolution profile of PQ SMEDDS in comparison to its commercial formulation in $0.01 \mathrm{~N} \mathrm{HCl}$ and buffer pH 6.8 is shown in Figure 3. The dissolution profile of PQ SMEDDS in various dissolution media showed similarity to the marketed formulation, $100 \%$ of $\mathrm{PQ}$ was released within 20 min irrespective of the $\mathrm{pH}$ of the dissolution medium.

\section{Stability studies}

Stability of PQ SMEDDS was determined by subjecting the formulation to accelerated temperature and humidity conditions and analysing the content of $\mathrm{PQ}$ at various intervals for a period of 6 months. No significant change in the PQ content was observed at the end of 3 months at all storage conditions. But the PQ content was decreased by $5 \%$ at $40^{\circ} \mathrm{C} / 75 \% \mathrm{RH}$ by the end of 6 months (Table 7). No significant 
Citation: Chudiwal P, Lahoti S (2018) Solid Self-Microemulsifying Drug Delivery System (SMEDDS) of Primaquine: Bio-distribution and Enhanced Liver Uptake. J Nanomed Nanotechnol 9: 483. doi: 10.4172/2157-7439.1000483

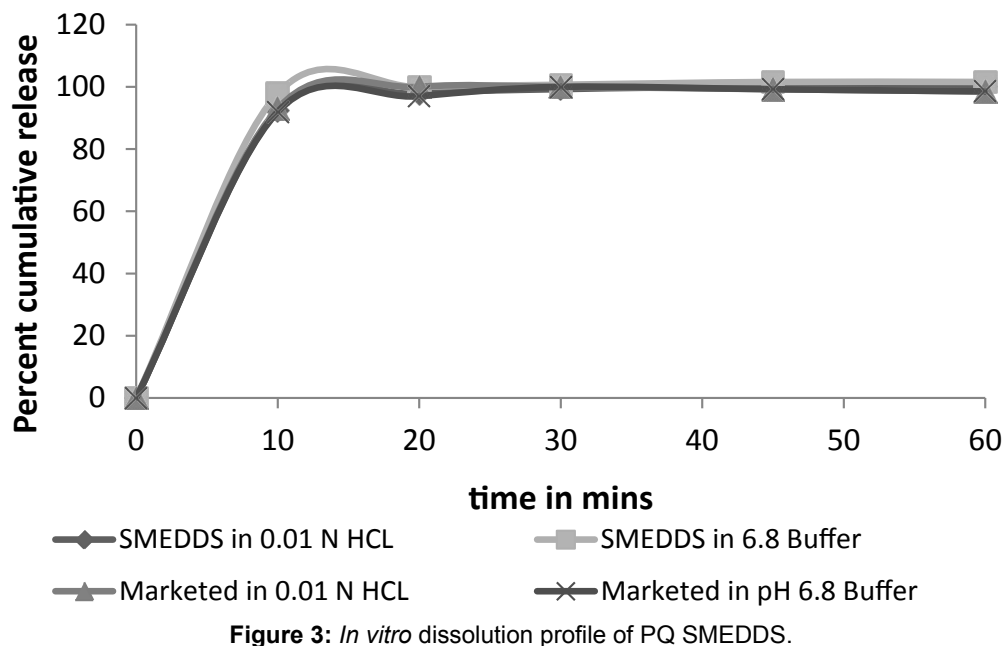

Figure 3: In vitro dissolution profile of $P Q$ SMEDDS.

\begin{tabular}{|c|c|c|c|c|c|c|c|c|c|c|}
\hline \multirow{2}{*}{$\begin{array}{c}\text { Storage } \\
\text { Condition }\end{array}$} & \multicolumn{9}{|c|}{ Percent PQ Content } & \multicolumn{3}{c|}{ Globule Size (nm) } \\
\cline { 2 - 10 } & Initial & 1 Month & 2 Month & 3 Month & 6 Month & Initial & 1 Month & 2 Month & 3 Month & 6 Month \\
\hline $25^{\circ} \mathrm{C} / 60 \%$ RH & $99.01 \pm 2.15$ & $99.51+1.98$ & $99.0 \pm 3.35$ & $99.12 \pm 2.95$ & $98.01 \pm 1.90$ & $75^{\mathrm{a}}(0.358)^{\mathrm{b}}$ & $74(0.317)$ & $80(0.351)$ & $85(0.375)$ & $87(0.372)$ \\
\hline $30^{\circ} \mathrm{C} / 65 \%$ RH & $99.01 \pm 2.15$ & $99.83 \pm 1.94$ & $98.97 \pm 2.24$ & $98.11 \pm 1.56$ & $97.25 \pm 1.22$ & $75(0.358)$ & $76(0.322)$ & $79(0.387)$ & $84(0.398)$ & $88(0.355)$ \\
\hline $40^{\circ} \mathrm{C} / 75 \%$ RH & $99.01 \pm 2.15$ & $98.73 \pm 1.11$ & $98.71 \pm 3.24$ & $97.25 \pm 3.56$ & $94.89 \pm 2.21$ & $75(0.358)$ & $75(0.364)$ & $82(0.379)$ & $87(0.401)$ & $88(0.362)$ \\
\hline
\end{tabular}

aparticle size expressed as mean $(n=3)$ where relative standard deviation was $<10 \%$.

${ }^{b}$ Polydispersity Index; Data were expressed as mean $(n=3)$.

Table 7: PQ content and particle size analysis of PQ SMEDDS at various storage conditions.

change in the particle size of the PQ SMEDDS was observed during the stability studies (Table 7). Furthermore, no change in other parameters such as physical properties, dissolution profile, was observed for the PQ SMEDDS (data not shown).

\section{In vivo efficacy studies}

In vivo antimalarial efficacy of the PQ SMEDDS was determined using the murine malaria model. We tested two different doses of PQ SMEDDS and compared them with equivalent doses of the marketed formulation. The control group that had no treatment and the group treated with placebo SMEDDS showed the highest parasitemia (Figure 4). All the other groups showed a significant reduction in parasitemia compared to the control group $(\mathrm{p}<0.05)$. SMEDDS 15 mg dosage showed highest antimalarial activity as compared to all other groups $(\mathrm{p}<0.05)$. The antimalarial activity of SMEDDS $7.5 \mathrm{mg}$ was statistically indistinguishable from PQ Suspension $15 \mathrm{mg}$ (Figure 4). On day 8, PQ SMEDDS $15 \mathrm{mg}$ showed around 50 percent activity which was 2-fold higher than that of the marketed formulation (Figure 5). Dunnett's post-test confirmed that at the dose level of $15 \mathrm{mg}$, the percent antimalarial activity of PQ-SMEDDS was significantly greater than that of the marketed formulations. As evident from the survival plot (Figure 6), SMEDDS $15 \mathrm{mg}$ also helped in prolonging the survival of the animals indicating protection for a longer duration. Thus PQ SMEDDS is effective in conferring anitmalarial protection at half the dosage of the marketed formulation.

\section{Biodistribution studies}

Biodistribution of PQ SMEDDS was determined in male Swiss mice following oral administration of fluorescently labelled formulation and using flow cytometry. The biodistribution of PQ SMEDDS and PQ suspension in liver is depicted in Figure 7. As observed from Figure 7 , oral administration of SMEDDS resulted in a significantly higher

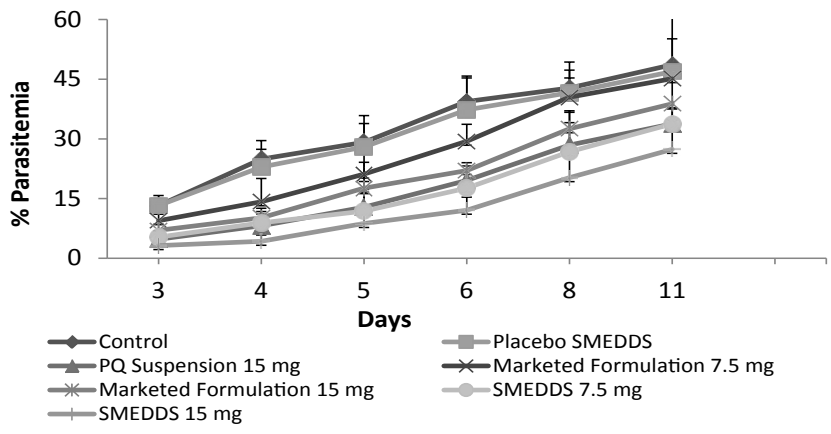

Figure 4: Percent parasitemia of $P Q$ SMEDDS.

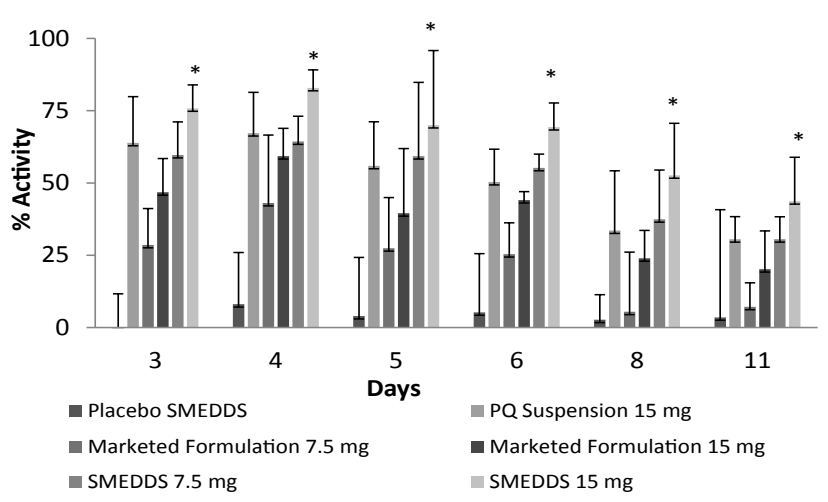

Figure 5: Percent activity of $P Q$ SMEDDS. All values are expressed as Mean $\pm \mathrm{SD}, \mathrm{n}=8$, For statistical significance, One-way ANOVA followed by Dunnett's test at ${ }^{*} p<0.05$ is applied wherein all treated groups are compared with $P Q$ marketed formulation group. 
Citation: Chudiwal P, Lahoti S (2018) Solid Self-Microemulsifying Drug Delivery System (SMEDDS) of Primaquine: Bio-distribution and Enhanced Liver Uptake. J Nanomed Nanotechnol 9: 483. doi: 10.4172/2157-7439.1000483
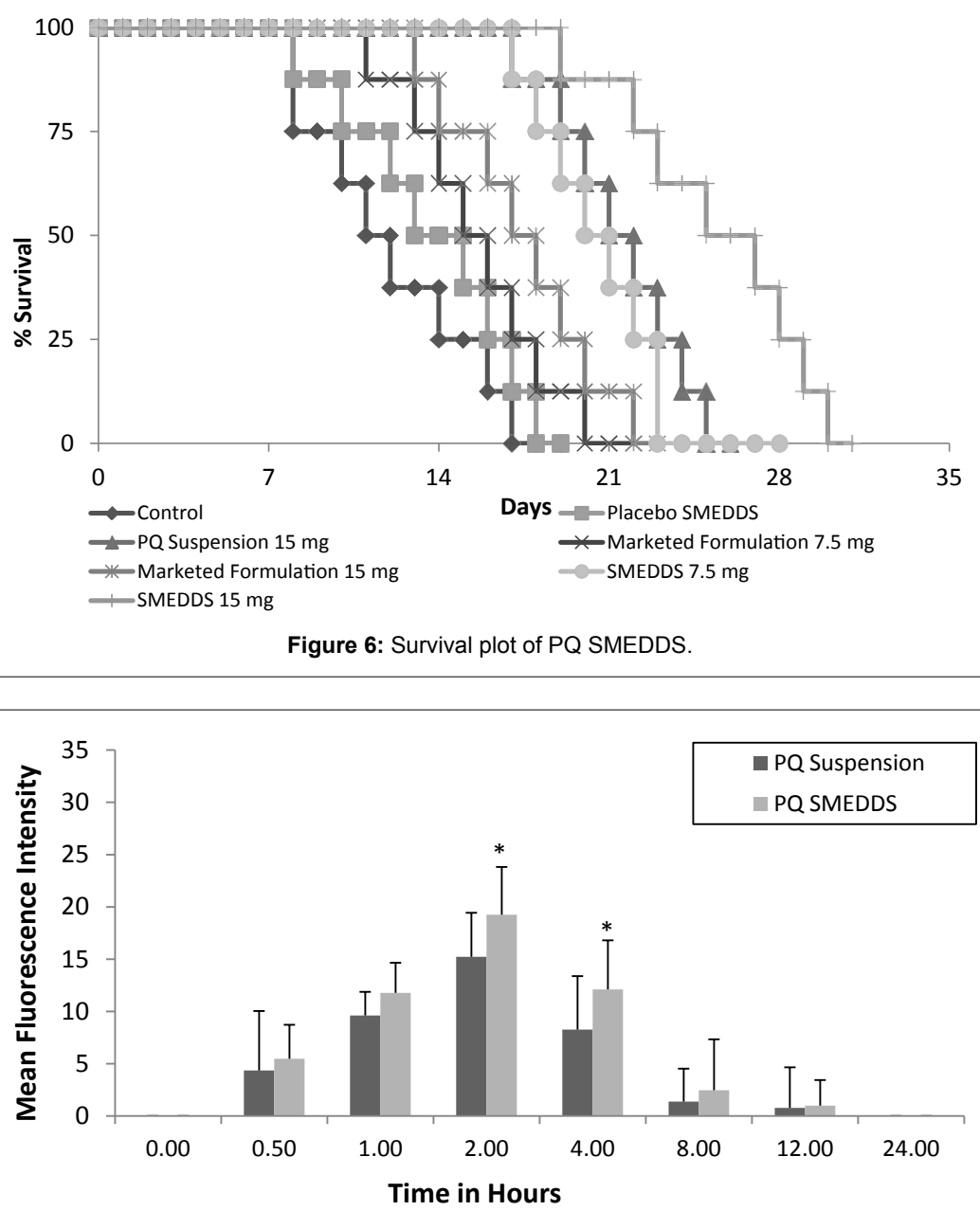

Figure 7: Biodistribution of $P Q$ SMEDDS and $P Q$ suspension in liver. All values are expressed as Mean $\pm S D$, $n=3$, For statistical significance, One-way ANOVA followed by Dunnett's test at ${ }^{*} p<0.05$ is applied wherein PQ SMEDDS group is compared with PQ suspension group.

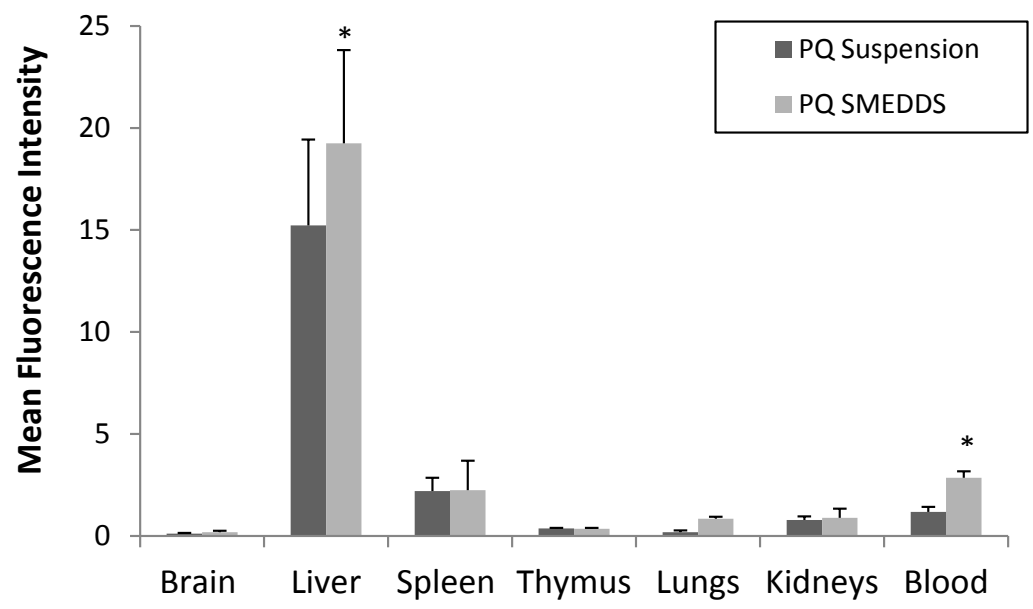

Figure 8: Biodistribution of $P Q S M E D D S$ and $P Q$ suspension in various organs at $2 \mathrm{~h}$. All values are expressed as Mean $\pm S D$, $n=3$, For statistical significance, Oneway ANOVA followed by Dunnett's test at ${ }^{*} p<0.05$ is applied wherein PQ SMEDDS group is compared with PQ suspension group.

concentration of $\mathrm{PQ}$ in the liver upto $4 \mathrm{~h}$ as compared to the PQ suspension group. $2 \mathrm{~h}$ after the administration, liver had the highest uptake, followed by the spleen and blood (Figure 8). An essentially similar profile of uptake was observed at other time points as well.
PQ SMEDDS resulted in a higher concentration of PQ in blood as compared to the PQ suspension. This suggests a faster absorption from SMEDDS than from suspension. The main advantage of PQ as an important antimalarial agent is because of its effectiveness against 
Citation: Chudiwal P, Lahoti S (2018) Solid Self-Microemulsifying Drug Delivery System (SMEDDS) of Primaquine: Bio-distribution and Enhanced Liver Uptake. J Nanomed Nanotechnol 9: 483. doi: 10.4172/2157-7439.1000483

Page 9 of 9

latent hepatic (hypnozoite) forms of P. vivax and also because of its gametocidal activity. Earlier reports of targeting PQ have been obtained after intravenous administration and show enhanced uptake in the liver. It is likely that SMEDDS after oral administration can enhance the drug uptake by liver because of enhanced bioavailability. Achieving higher concentrations of PQ at its site of action, i.e., liver can enhance its efficacy thus help in reducing relapses caused by the hepatic forms of the parasite.

\section{Conclusion}

SMEDDS of PQ with a size range of 75-80 nm were successfully formulated and stabilized by adsorbing onto an inert, solid adsorbent, Aerosil 200. Accelerated stability studies indicated that the developed PQ SMEDDS were stable for a period of 6 months at all storage conditions. Effective antimalarial activity at $50 \%$ reduced dose was achieved with SMEDDS as compared with the marketed formulation after oral administration. Improved therapeutic efficacy of PQ may be attributed to enhanced oral bioavailability. Biodistribution studies show that the greatest concentration of the drug is targeted to the liver. This could prove especially beneficial as PQ is especially useful against the hepatic hypnozoite forms of the parasite. Thus, SMEDDS can be regarded as novel and commercially feasible alternative to current PQ formulations to treat latent stage $P$. vivax malaria and reduce toxicity.

\section{Future Perspective}

The work published so far is for evaluating the efficacy of PQ formulations in murine malaria models use $P$. berghei ANKA strain parasite. However this strain of parasite does not develop latent hepatic forms. Hence a validated animal model with latent hepatic forms should be further developed to understand the therapeutic efficacy of the drugs acting on liver stages. Furthermore, it would also be worthwhile to evaluate short-term and long-term toxicity of the formulations after oral administration.

\section{Acknowledgements}

Authors are thankful to IPCA Laboratories (Mumbai, India) for the gift sample of Primaquine. The authors would also like to thank BASF (Mumbai), India, Gattefosse (France), Associated Capsules Group, Mumbai, India -Asia Pvt. Ltd. (Mumbai, India), Colorcon Asia Mumbai, India and Gangwal Chemicals (Mumbai, India) for providing gift samples of various excipients.

\section{References}

1. Annie MD, Zhengrong $C$ (2005) Targeting Primaquine into liver using chylomicron emulsions for potential vivax malaria therapy. Int J Pharm 303 $143-152$.

2. Kamalinder KS, Sharvani KV (2008) Formulation, antimalarial activity and biodistribution of oral lipid nanoemulsion of primaquine. Int J Pharm 347: 136-143.

3. Sasithon P, Kesinee C, Arun C, Ralf C, Sornchai L, et al. (2004) Activities of Artesunate and Primaquine against asexual- and sexual-stage parasites in falciparum malaria. Antimicrob Agents Chemother 48: 1329-1334.

4. Jeanne MS, Lynne SG, David MG, David AH (2005) Diagnostic and therapeutic pitfalls associated with Primaquine-tolerant Plasmodium vivax. J Clin Microbiology 43: 978-981.

5. Laura JC, Bolchoz, Robert AB, David CM, David JJ (2001) Primaquine-induced hemolytic anemia: formation and hemotoxicity of the arylhydroxylamine metabolite 6-Methoxy-8-hydroxylaminoquinoline. J Pharmacol Exp Ther 297: 509-515.

6. Nuno V, Rui M, Paula G (2009) Primaquine revisited six decades after its discovery. Eur J Med Chem 44: 937-953.

7. Hill DR, Baird JK, Parise ME, Lewis LS, Ryan ET, et al. (2006) Primaquine: report from $C D C$ expert meeting on malaria chemoprophylaxis I. American Journal of Tropical Medicine and Hygiene 75: 402-415.

8. Bhadra D, Yadav AK, Bhadra S, Jain NK (2005) Glycodendrimeric nanoparticulate carriers of Primaquine Phosphate for liver targeting. In J Pharm 295: 221-233.

9. Mayorga P, Puisieux F, Couarraze G (1996) Formulation study of a transdermal delivery system of Primaquine. Int J Pharm 132: 71-79.

10. Labhasetwar VD, Dorle AK (1990) Nanoparticles - A colloidal drug delivery system for Primaquine and Metronidazole. J Control Release 12: 113-119.

11. Borahde V, Nair H, Hegde D (2008) Design and evaluation of selfmicroemulsifying drug delivery system (SMEDDS) of Tacrolimus. AAPS Pharm Sci Tech 9: 13-20.

12. Maulik JP, Natvarlal MP, Madhabhai MP (2010) A self-microemulsifying drug delivery system (SMEDDS). Int J Pharm Sci 4: 29-35.

13. United States Pharmacopoeial convention (2003) United States Pharmacopoeia Asian Edition, USP 27. Rockville, MD, USA.

14. Peters W, Portus JH, Robinson BL (1975) The chemotherapy of rodent malaria. $X X I I$. The value of drug-resistant strains of $P$. berghei in screening of blood schizonticidal activity. Ann Trop Med Parasitol 69: 155-171.

15. Fidlock DA, Rosenthal PJ, Croft SL, Brun R, Nwaka S (2008) Antimalarial efficacy Screening: in vitro and in vivo protocols, Supplemental file, Antimalaria drug Discovery: efficacy model for compound screening supplementary document. 